\title{
Prurigo pigmentosa with new-onset diabetic ketoacidosis
}

\author{
Jérôme Coulombe MD, Darosa Lim MD, Louis Geoffroy MD
}

Cite as: CMAJ 2021 November 29;193:E1815. doi: 10.1503/cmaj.210723

A 13-year-old, previously healthy, boy presented to the emergency department with a 1-week history of progressive, highly pruritic urticarial erythematous papules and confluent plaques in a symmetric, reticulated distribution on his neck, back and torso (Figure 1). Older lesions were hyperpigmented and squamous.

The patient reported a progressive, 1-month history of $6 \mathrm{~kg}$ weight loss, polydipsia, polyuria, polyphagia and fatigue. Laboratory results showed hyperglycemia, ketonemia, glycosuria and metabolic acidosis, consistent with diabetic ketoacidosis. Our differential diagnoses for the skin lesions were prurigo pigmentosa, contact dermatitis, urticaria, confluent and reticulated papillomatosis (i.e., Gougerot-Carteaud syndrome) and erythema ab igne. We biopsied the skin, which showed subacute spongiosis, with eosinophilic and lymphohistiocytic perivascular infiltrates and papillary edema. We diagnosed prurigo pigmentosa.

As per our local protocol for diabetic ketoacidosis, we prescribed intravenous, then subcutaneous insulin, which led to adequate control of glucose and ketone levels, and improvement of the skin lesions over 3 weeks (Appendix 1, available at www. cmaj.ca/lookup/doi/10.1503/cmaj.210723/tab-related-content).

Prurigo pigmentosa is an inflammatory dermatitis with more than 300 cases reported. ${ }^{1}$ Its exact cause remains unknown; however, proketogenic states contribute to the pathogenesis, leading to an accumulation of ketone bodies around the blood vessels that may result in dermal perivascular inflammation. ${ }^{1,2}$

Prurigo pigmentosa has been reported with intense fasting, anorexia nervosa, hyperemesis gravidarum, bariatric surgery, type I diabetes mellitus and, more recently, ketogenic diets. ${ }^{1,3}$ Risk factors for development or worsening of lesions include dietary changes, friction, sweat, ketonuria and hormonal changes. ${ }^{3}$ The lesions are pruritic and usually present with erythematous reticulated papules and plaques on the back, chest and neck areas, sparing the face, arms, legs and mucosa. Mottled hyperpigmentation occurs as lesions resolve. ${ }^{1}$ Diagnosis of prurigo pigmentosa is largely clinical, as histologic findings are nonspecific and can vary depending on the lesions' stages; different stages can coexist. In our patient, histological results were consistent with clinical findings of lesions at different stages. Although not a classic finding, a predominance of eosinophils has been reported with prurigo pigmentosa, and necrotic keratinocytes are not always present. ${ }^{3,4}$

Treatment is tailored to the cause of the proketogenic states. Specialists may prescribe anti-inflammatory adjunct therapies if symptoms do not resolve, including tetracycline or macrolide antibiotics, isotretinoin, dapsone, colchicine or phototherapy. ${ }^{1}$

\section{References}

1. Beutler BD, Cohen PR, Lee RA. Prurigo pigmentosa: literature review. Am J Clin Dermatol 2015;16:533-43.

2. Ohnishi T, Kisa H, Ogata E, et al. Prurigo pigmentosa associated with diabetic ketoacidosis. Acta Derm Venereol 2000;80:447-8.

3. Mufti A, Mirali S, Abduelma A, et al. Clinical manifestations and treatment outcomes in prurigo pigmentosa (Nagashima disease): a systematic review of the literature. JAAD Int 2021;3:79-87.

4. Böer A, Misago N, Wolter M, et al. Prurigo pigmentosa: a distinctive inflammatory disease of the skin. Am J Dermatopathol 2003;25:117-29.

\section{Competing interests: None declared.}

This article has been peer reviewed.

The authors have obtained patient consent.

Affiliations: Departments of Pediatrics (Coulombe, Geoffroy, Lim), CHU Sainte-Justine, Montréal, Que.
Content licence: This is an Open Access article distributed in accordance with the terms of the Creative Commons Attribution (CC BY-NC-ND 4.0) licence, which permits use, distribution and reproduction in any medium, provided that the original publication is properly cited, the use is noncommercial (i.e., research or educational use), and no modifications or adaptations are made. See: https://creativecommons.org/licenses/by-nc-nd/4.0/

Correspondence to: Jérôme Coulombe, jerome.coulombe@umontreal.ca 